\title{
BMJ Open Lumbopelvic pain, anxiety, physical activity and mode of conception: a prospective cohort study of pregnant women
}

Emeline Lardon, ${ }^{1,2}$ Audrey St-Laurent, ${ }^{1}$ Véronique Babineau, ${ }^{3}$ Martin Descarreaux, ${ }^{1}$ Stephanie-May Ruchat ${ }^{1}$

To cite: Lardon E, St-Laurent A, Babineau V, et al. Lumbopelvic pain, anxiety, physical activity and mode of conception: a prospective cohort study of pregnant women. BMJ Open 2018;8:e022508. doi:10.1136/ bmjopen-2018-022508

- Prepublication history for this paper is available online. To view these files, please visit the journal online (http://dx.doi org/10.1136/bmjopen-2018022508).

Received 28 February 2018 Revised 28 September 2018 Accepted 4 October 2018

A) Check for updates

(c) Author(s) (or their employer(s)) 2018. Re-use permitted under CC BY-NC. No commercial re-use. See rights and permissions. Published by BMJ.

${ }^{1}$ Department of Human Kinetics, Université du Québec à TroisRivières, Trois-Rivieres, Quebec, Canada

${ }^{2}$ Institut Franco-Européen de Chiropraxie, Paris, France

${ }^{3}$ Department of Obstetrics and Gynaecology, Centre intégré universitaire de santé et de services sociaux de la Mauricieet-du-Centre-du-Québec, affiliated to the University of Montreal, Trois-Rivières, Quebec, Canada

Correspondence to

Dr Stephanie-May Ruchat; stephanie-may.ruchat@uqtr.ca

\section{ABSTRACT}

Objectives Pregnancy-related lumbopelvic pain (LPP) is a frequent condition known to significantly affect women in their daily life. The aetiology of pregnancy-related LPP pain is still not clearly established but the mode of conception has been suggested to contribute to LPP. Anxiety related to fertility treatments may be one of the contributing factors. The primary objectives of this study were to determine the evolution of LPP prevalence and severity, and anxiety throughout pregnancy in women who conceived spontaneously (SP) or after fertility treatments (FT). A further aim was to examine the relationship between pregnancy-related LPP severity and anxiety. The secondary objective was to determine the evolution of physical activity and their correlation with the severity of pregnancy-related LPP.

Design Prospective cohort study.

Setting Pregnant women were recruited through physicians' referrals, posters and newspaper advertisements in the local and surrounding communities (hospital, maternity care clinic, prenatal centres, sports centres, local university) in the city of Trois-Rivières, Canada.

Participants 59 pregnant women (33 SP and 26 FT) were assessed during the first, second and third trimester of pregnancy.

Primary and secondary outcome measures Pregnancyrelated LPP prevalence and severity (primary), trait and state anxiety, and physical activity levels (secondary). Results There was no relationship between the mode of conception and the outcome measures. The prevalence and severity of LPP increased over the course of pregnancy (time effect, $p<0.0001$ ) whereas trait anxiety decreased from early to mid-pregnancy (time effect, $p=0.03$ ). Activity limitations increased throughout pregnancy (time effect, $p<0.0001$ ) and physical activity levels decreased (time effect, $p<0.0001$ ). The severity of LPP was positively correlated with activity limitations ( $r=0.51$ to 0.55$)$ but negatively with physical activity levels ( $r=-0.39$ to -0.41$)$.

Conclusions Maternal health-related factors, such as LPP, anxiety and physical activity, are not different in women who conceived spontaneously or after fertility treatments. The more LPP was severe, the more the women were physically limited and inactive.
Strengths and limitations of this study

- This is a prospective cohort study of pregnant women who were assessed at each trimester of pregnancy, allowing to determine the evolution of several maternal health-related factors that are known to change over the course of pregnancy

- Primary and secondary outcomes were collected using validated tools

- The low number of women who achieved a pregnancy following in vitro fertilisation prevented us to fully test our hypotheses; thus larger studies are needed to better understand whether in vitro fertilisation contributes to pregnancy-related LPP

- More than half of the participants had a university degree, which is not representative of our local population. The results may therefore not be broadly generalisable

\section{INTRODUCTION}

More than $50 \%$ of women experience pain in the lumbopelvic area during pregnancy. ${ }^{1}$ Low back pain (LBP) is defined as pain localised below the ribs, but above the gluteal folds, with or without radiation down the legs, ${ }^{2}$ whereas pelvic girdle pain (PGP) is defined as pain 'experienced between the posterior iliac crest and the gluteal fold, particularly in the vicinity of the sacroiliac joints. The pain may radiate in the posterior thigh and can also occur in conjunction with/or separately in the symphysis'. 1 The term lumbopelvic pain (LPP) is used when no distinction is made between PGP and LBP. ${ }^{3}$ Thus the wide range of cases in the reported prevalence of LPP in the literature $(45 \%-73 \%)^{45}$ has been attributed to the different criteria used to classify types and severity of pain, and the different periods during pregnancy LPP was assessed. The onset of LPP varies considerably, between the end of the 
Table 1 Baseline characteristics of the 59 pregnant women included in study

\begin{tabular}{|c|c|c|c|}
\hline Variables & FT $(n=26)$, mean $\pm S D$ & SP $(n=33)$, mean $\pm S D$ & P values \\
\hline Fertility treatments & $\begin{array}{l}\mathrm{OS}=7 \\
\mathrm{IUI}=12 \\
\mathrm{IVF}=7\end{array}$ & - & \\
\hline Age (years) & $32.2 \pm 3.6$ & $30.9 \pm 4.2$ & 0.23 \\
\hline Parity & $0.4 \pm 0.6$ & $0.6 \pm 0.6$ & 0.36 \\
\hline $0(\mathrm{n})$ & $57.7 \%(15)$ & $45.5 \%(15)$ & 0.35 \\
\hline Prepregnancy BMI (kg/cm²) & $26.3 \pm 7.3$ & $25.2 \pm 6.6$ & 0.54 \\
\hline Underweight $<18.4$ & $0 \%(0)$ & $3.1 \%(1)$ & 0.81 \\
\hline Normal weight (18.5-24.9) & $60.0(15)$ & $62.5 \%(20)$ & \\
\hline Overweight (25.0-29.9) & $20.0(5)$ & $18.8 \%(6)$ & \\
\hline University degree & $57.7 \%(15)$ & $66.7 \%(22)$ & \\
\hline LPP history (yes)* & $46.2 \%(12)$ & $54.6 \%(18)$ & 0.52 \\
\hline $\begin{array}{l}\text { Prevalence of pregnancy-related LPP over the last } \\
\text { week (yes) }\end{array}$ & $34.6 \%(9)$ & $48.5 \%(16)$ & 0.33 \\
\hline Severity of pregnancy-related LPP over the last week & $2.9 \pm 1.9$ & $4.1 \pm 2.3$ & 0.18 \\
\hline State anxiety & $37.4 \pm 11.6$ & $34.2 \pm 9.1$ & 0.28 \\
\hline Trait anxiety & $39.8 \pm 10.0$ & $37.1 \pm 9.4$ & 0.26 \\
\hline Daily steps & $5328 \pm 1551$ & $5569 \pm 1552$ & 0.8 \\
\hline Daily MVPA (min) & $16.3 \pm 10.0$ & $17.4 \pm 13.2$ & 0.97 \\
\hline
\end{tabular}

Missing data: prepregnancy BMI: 1 FT, 1 SP; state and trait anxiety: 1 SP; accelerometer data: 4 SP; 6 FT

*LPP history includes history of pregnancy-related LPP and LPP not related to pregnancy.

BMI, body mass index; FT, fertility treatment; IUI, intrauterine insemination; IVF, in vitro fertilisation; LPP, lumbopelvic pain; MVPA, moderateto-vigorous physical activity; OS, ovarian stimulation; SP, spontaneous conception.

first trimester to the first month post-delivery, with a peak of symptoms generally occurring between the 24th and 36th weeks of pregnancy. ${ }^{6}$ Pregnancy-related LPP is a debilitating condition that is known to affect women's quality of life, ${ }^{7}$ with repercussions such as disruption of sleep, increased psychological stress, social and sexual life and work capacity. ${ }^{4-10}$ Pregnant women experiencing LPP are also known to be less physically active during pregnancy. ${ }^{11}$ Prenatal physical activity is an important component of a healthy pregnancy ${ }^{12}$ and all women without contraindication to exercise are encouraged to be regularly active throughout pregnancy to benefit from it. ${ }^{13}{ }^{14}$ On the other hand, pregnancy-related LPP can contribute to maternal physical inactivity and its associated maternal, fetal and neonatal complications. ${ }^{12}$

Several factors are believed to be involved in pregnancy-related LPP development, such as degenerative metabolic, genetic, hormonal and biomechanical factors and non-optimal joint stability. ${ }^{16}$ Another factor of interest is the mode of conception, in other words, naturally or after fertility treatments. A study reported that pregnant women who underwent in vitro fertilisation (IVF) treatments had a two times higher prevalence rate of sacral pain in early and late pregnancy, as well as a higher frequency of positive results on pelvic pain provocation tests in late pregnancy. ${ }^{15}$ The authors concluded that relaxin causes pelvic pain because relaxin is higher in IVF pregnancies. ${ }^{16}$ Psychosocial factors may also be involved in the development of LPP. Higher anxiety levels experienced in women who conceived after IVF might contribute to the higher pregnancy-related LPP prevalence observed in these women. As reported by a systematic review, women who conceived following fertility treatments had greater pregnancy-specific anxiety than those who conceived naturally. ${ }^{17}$ Based on a multi-centre study including 1158 women, higher levels of anxiety was reported to be among the most notable factors associated with a higher likelihood of reporting LBP. ${ }^{18}$ However, to the best of our knowledge, no study has examined pregnancy-related LPP among women who achieve pregnancy naturally or after fertility treatment, and whether anxiety is a contributing factor to the development of LPP. 


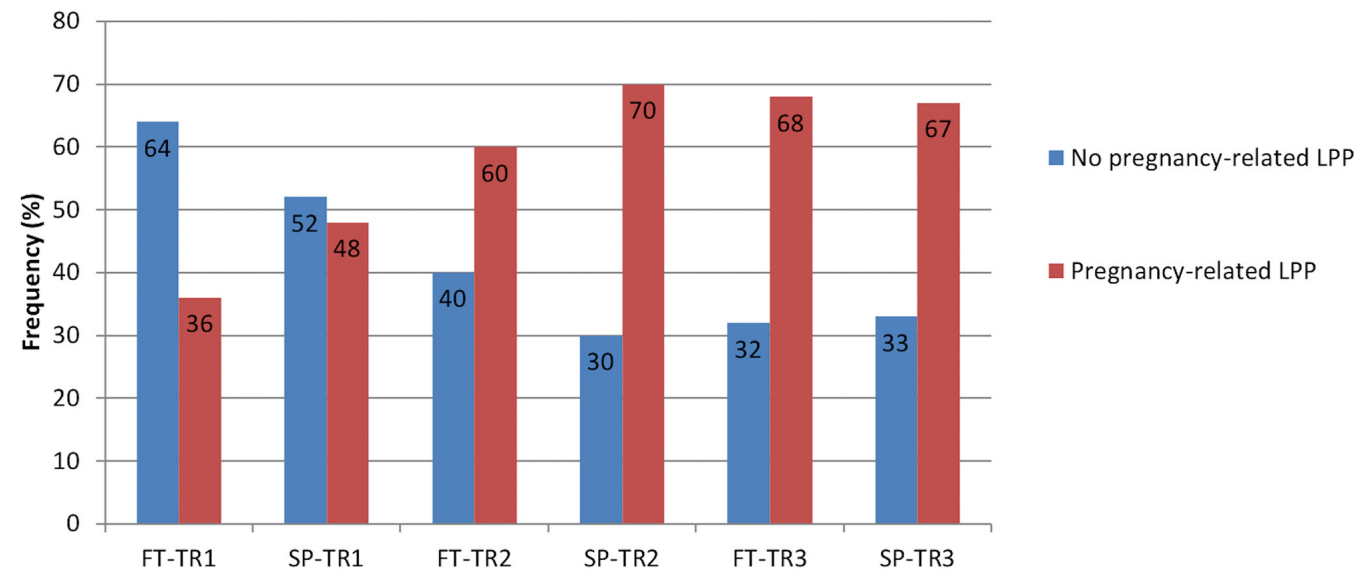

Figure 1 Prevalence of pregnancy-related lumbopelvic pain (LPP) in women who conceived spontaneously (SP) or after fertility treatments (FT) over the course of pregnancy. TR1: first trimester of pregnancy; TR2: second trimester of pregnancy; TR3: third trimester of pregnancy.

The primary objectives of this prospective cohort study were to determine the evolution of LPP prevalence and severity, as well as anxiety, over the course of pregnancy in women who conceived naturally or after fertility treatments, and to examine the possible relationship between pregnancy-related LPP severity and anxiety levels. As pregnancy-related LPP has a significant impact on women's daily life activities, the secondary objective of our study was to determine the evolution of physical activity behaviours throughout pregnancy and whether the severity of LPP was correlated to these factors. Our primary hypotheses are that LPP prevalence and severity, as well as anxiety levels will increase over the course of pregnancy but more strongly in women who conceived after fertility treatments, and that pregnancy-related LPP severity will be positively correlated with anxiety levels. As a result, our secondary hypotheses are that activity limitations will increase whereas physical activity behaviours will decrease over the course of pregnancy but more significantly in women who conceived after fertility treatments, and that the severity of pregnancy-related LPP will be positively correlated with activity limitations but negatively with physical activity behaviours.

\section{MATERIALS AND METHODS}

\section{Study design and selection of participants}

This is a prospective cohort study of pregnant women who were recruited between October 2015 and September 2016. Women who achieved a spontaneous pregnancy (SP group) and women who achieved pregnancy following fertility treatments (FT group) were recruited through referrals by physicians and clinic coordinators, posters and newspaper advertisements in the local and surrounding communities (hospital, maternity care clinic, prenatal centres, sports centres, local university) in the city of Trois-Rivières, Canada. Women under 14 weeks of gestation, with a singleton pregnancy and able

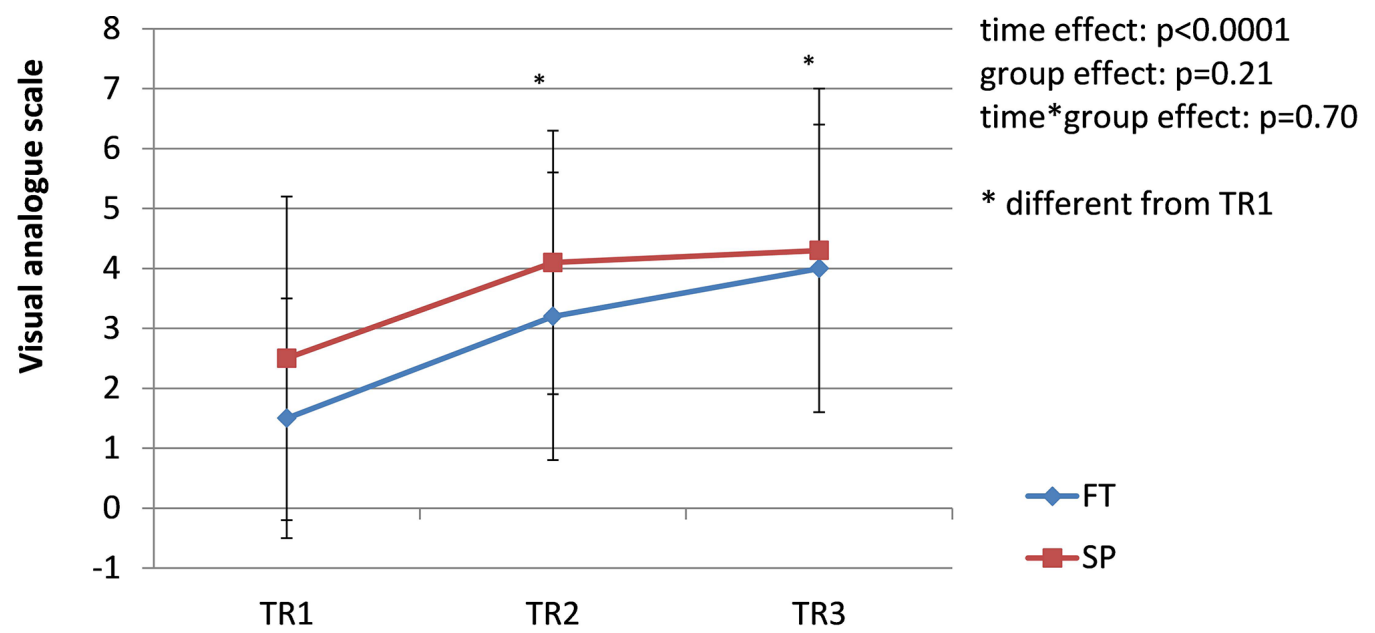

Figure 2 Evolution of pregnancy-related lumbopelvic pain (LPP) severity in women who conceived spontaneously (SP) or after fertility treatments (FT) over the course of pregnancy. TR1: first trimester of pregnancy; TR2: second trimester of pregnancy; TR3: third trimester of pregnancy. 


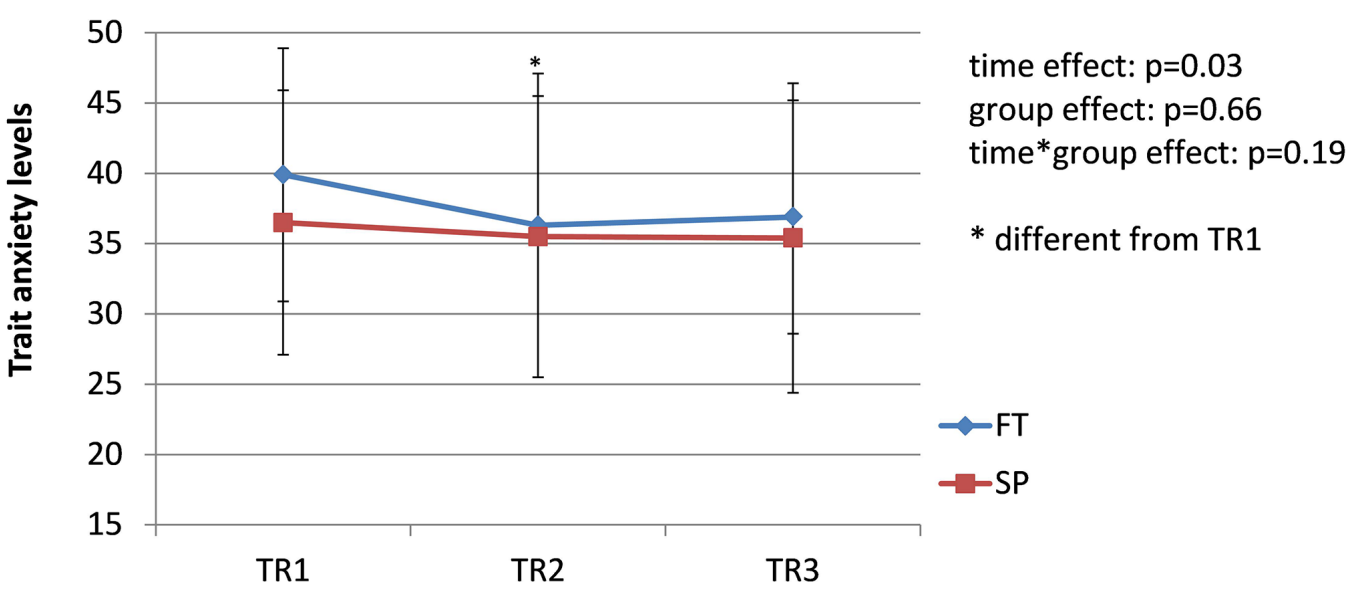

Figure 3 Evolution of trait anxiety in women who conceived spontaneously (SP) or after fertility treatments (FT) over the course of pregnancy. TR1: first trimester of pregnancy; TR2: second trimester of pregnancy; TR3: third trimester of pregnancy.

to understand, speak and write French were considered eligible to participate in the study. The study was approved by the local Research Ethics Committees (CER-2015-003 and CER-15-214-07.10) and all participants provided their written informed consent.

\section{Outcome measures and measurement tools}

Women were followed from the first trimester of pregnancy until delivery through three evaluations (first trimester [TR1]: 10-16 weeks, second trimester [TR2]: 24-28 weeks and third trimester [TR3]: 32-36 weeks of gestation). In each trimester, a member of the research team asked the women if they have had pregnancy-related LPP over the last 7 days or if they were having LPP presently using the illustration provided in the French version of the Pelvic Girdle Questionnaire (PGQ). ${ }^{19}$ If a woman had or was having pregnancy-related LPP, she was asked to rate pain intensity using a visual analogue pain scale. This scale is a self-reported measurement tool used by health professionals allowing the patient to rate pain from 0 (no pain) to 10 (extreme pain). ${ }^{20}$

The levels of anxiety was assessed during TR1, TR2 and TR3 using the French-Canadian version ${ }^{21}$ of the StateTrait Anxiety Inventory (STAI). ${ }^{22}$ The STAI is a self-reported questionnaire assessing the presence and severity of current symptoms of anxiety (state anxiety scale) and a generalised propensity to be anxious (trait anxiety scale). Each scale comprises 20 items rated with a 4-level Likert scale. The range of score for each scale is $20-80$, with the higher score indicating greater anxiety levels. The STAI has been widely used in research with pregnant women and it does reflect the anxiety-related experiences of pregnant women. Its use with pregnant women is therefore appropriate. ${ }^{23}$

Activity limitations and symptoms associated with pregnancy-related LPP were assessed in TR2 and TR3 using the completed the French-Canadian version of the PGQ was used. ${ }^{19}$ The PGQ is a condition-specific measure developed for pregnant and postpartum women. It consists of 20 activity items and five symptom items on a four-point response scale and assesses activity limitations and symptoms associated with pain in the lumbopelvic region. The range of score is $0 \%-100 \%$, with a higher score indicating greater activity limitations and symptoms. The PGQ is reliable and valid for both pregnant and postpartum women with pregnancy-related LPP. ${ }^{24}$

Finally, physical activity levels were objectively measured at each trimester of pregnancy using the ActiGraph GT3X (ActiGraph, Pensacola, Florida, USA), a

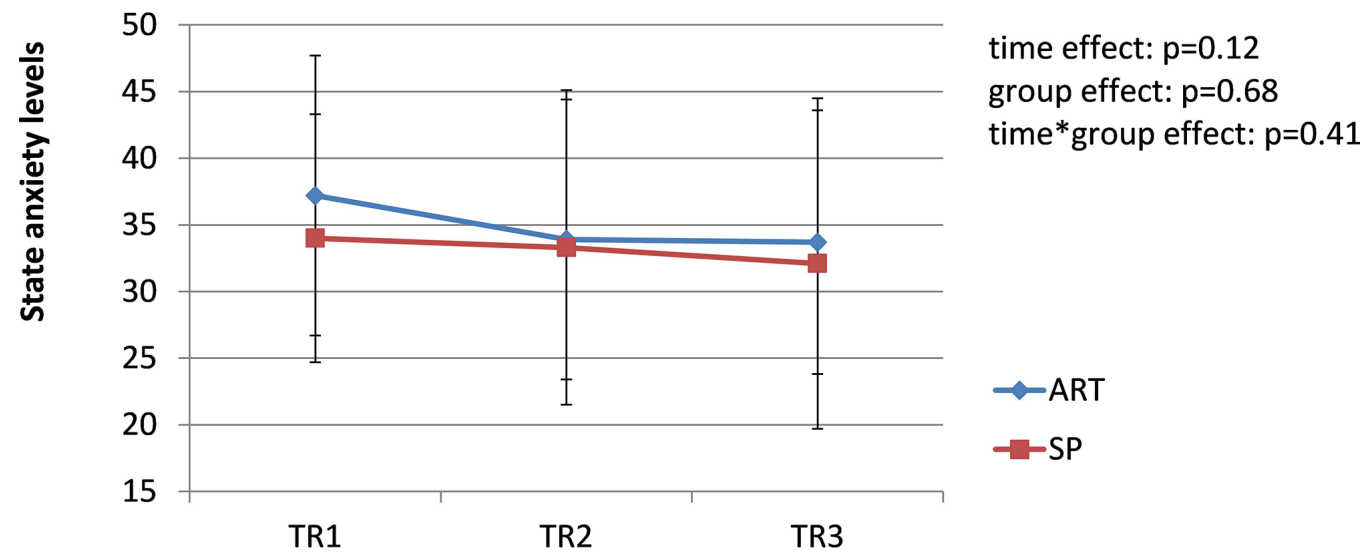

Figure 4 Evolution of state anxiety in women who conceived spontaneously (SP) or after fertility treatments (FT) over the course of pregnancy. TR1: first trimester of pregnancy; TR2: second trimester of pregnancy; TR3: third trimester of pregnancy. 


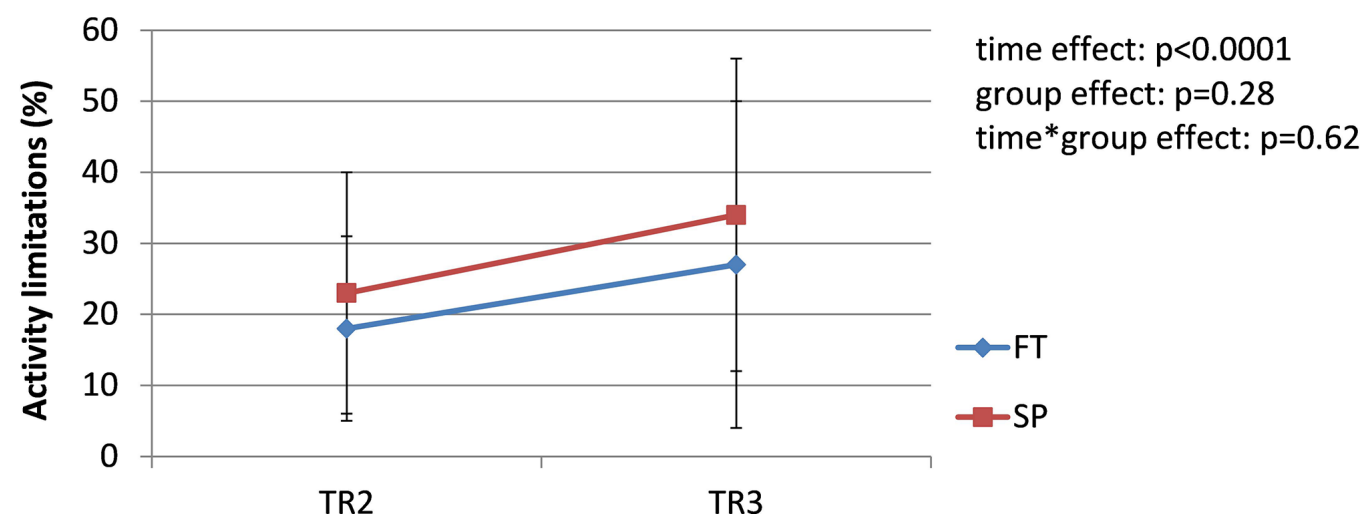

Figure 5 Evolution of activity limitations in women who conceived spontaneously (SP) or after fertility treatments (FT) over the course of pregnancy. TR1: first trimester of pregnancy; TR2: second trimester of pregnancy; TR3: third trimester of pregnancy.

triaxial accelerometer measuring data in a $60 \mathrm{~s}$ epoch. The women were instructed to wear the monitor over the hip on an elastic belt for 7 consecutive days from wake-up time to bedtime. They were allowed to remove the accelerometer when sleeping, showering or engaging in water activities. Furthermore, the women received a daily diary to document wear and non-wear time periods and water activities. According to the method used in the Canadian Health Measures Survey, valid data were defined as 4 days or more of monitoring for 10 hours or more of wear time per day. ${ }^{25}$ Pregnant women were encouraged to maintain their usual activities. Data were processed using the Actilife software V.6.13.2 (ActiGraph, Florida, USA). The accelerometer data obtained were averaged across valid wear days. To derive the activity frequency, intensity and duration of the measured activity in counts per minute per day, the Freedson equation was used: sedentary $(<100$ counts $)$, light (100-1951 counts), moderate (1952-5724), vigorous (5725-9498), and very vigorous $(>9498),{ }^{26}$ as previously used in pregnant women. ${ }^{27}$ Non-wear time was defined as a period of zero counts for $\geq 60$ consecutive minutes, admitting a maximum of two consecutive minutes between 1 and 100 counts $/ \mathrm{min}$. When a third observation was between 1 and 100 counts or one observation was more than 100 counts, the non-wear period was ended. Bouts of moderate-to-vigorous physical activity (MVPA) was defined as a minimum of 10 consecutive minutes above 1952 counts and ended with more than two consecutive records below this threshold.

\section{Patient and public involvement}

Patients and public were not involved in the design and conduct of this study. The results will not be disseminated to study participants.

\section{Statistical analysis}

Means and SD, as well as percentages, were computerised for variables of interest. Student's t-test was used to compare socio-demographic and anthropometric characteristics between SP and FT women. For categorical variables, the $\chi^{2}$ test was used. The MIXED procedure of SAS software was used to test the effect of time (trimesters), group (SP and FT women) and potential interaction effects on the outcome measures (ie, the severity of pregnancy-related LPP and anxiety levels (objective 1), and physical activity behaviours (objective 2)). The assumption of sphericity was tested using Mauchly's Test of Sphericity. Variables

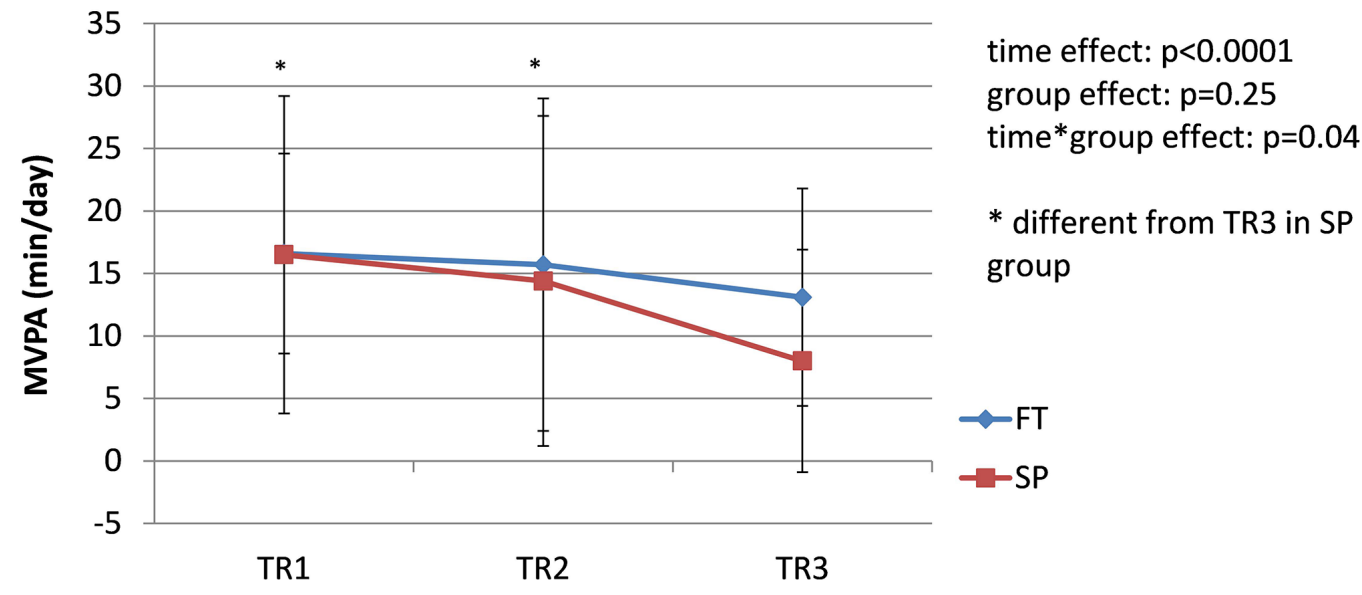

Figure 6 Evolution of daily moderate-to-vigorous physical activity (MVPA) in women who conceived spontaneously (SP) or after fertility treatments (FT) over the course of pregnancy. TR1: first trimester of pregnancy; TR2: second trimester of pregnancy; TR3: third trimester of pregnancy. 


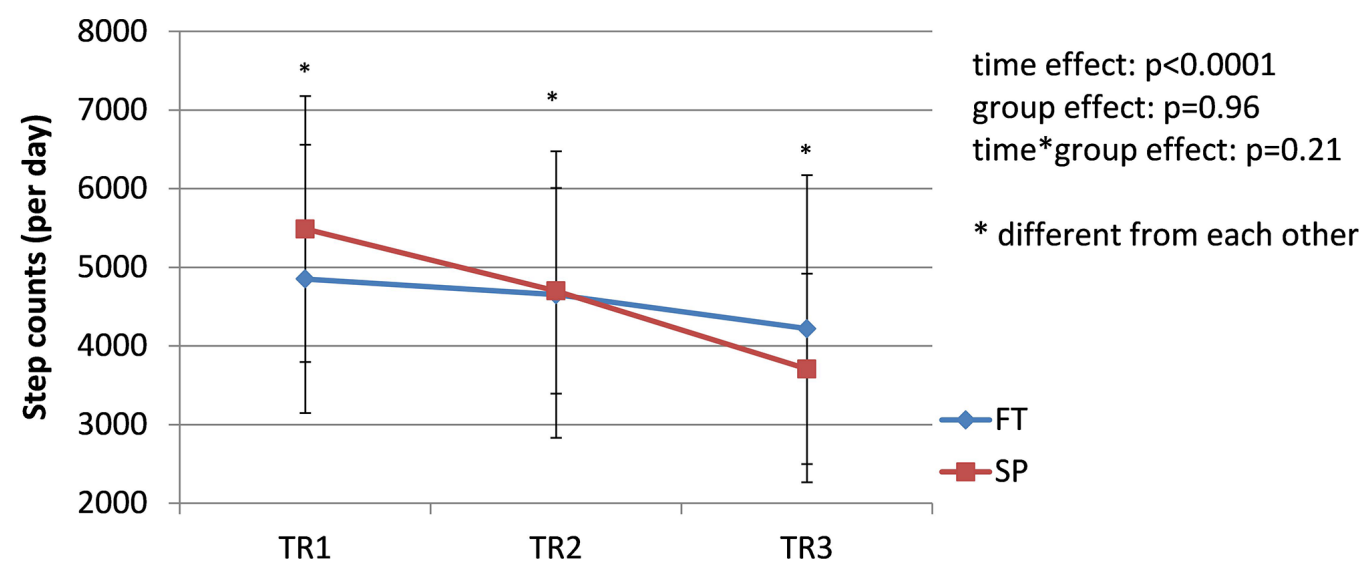

Figure 7 Evolution of daily step counts in women who conceived spontaneously (SP) or after fertility treatments (FT) over the course of pregnancy. TR1: first trimester of pregnancy; TR2: second trimester of pregnancy; TR3: third trimester of pregnancy.

that did not meet the sphericity assumption were analysed following a Geisser Greenhouse correction. When a significant effect of time, group or interaction effect was found, post-hoc analyses were conducted using the Tukey test. To test whether the severity of pregnancy-related LPP was correlated to the levels of anxiety (objective 1), and physical activity behaviours (objective 2) at each trimester of pregnancy, Pearson's correlation analyses were used. Statistical analyses were performed by using the SAS software V.9.4 and the level of significance was set to $p$ value $\leq 0.05$.

\section{RESULTS}

Between October 2015 and September 2016, the study was presented by physicians to 117 eligible pregnant women, among which 62 women accepted to participate. Reasons for not agreeing to participate to the study were lack of interest or lack of time. Three women (one in SP group and two in FT group) were excluded due to loss to follow-up $(\mathrm{n}=1)$, miscarriage $(\mathrm{n}=1)$ or missing data $(\mathrm{n}=1)$, leaving 59 women (33 SP and 26 FT) for the statistical analyses.

The characteristics of pregnant women are presented in table 1. No significant difference in socio-demographic and pre-pregnancy anthropometric characteristics was found between the groups $(p>0.05)$. Women were on average in their early thirties and approximately half of them were nulliparous. More than half were of normal weight pre-pregnancy (body mass index $18.5-24.9 \mathrm{~kg} / \mathrm{m}^{2}$ ) and had a university degree. Women's LPP history, related or not to a previous pregnancy, was also similar between the groups, with approximately $50 \%$ of the women reporting a history of LPP (table 1). Finally, the prevalence and severity of pregnancy-related LPP, anxiety and physical activity levels were not different between SP and FT pregnant women at study entry (table 1). Data showed that on average, women considered LPP as moderate

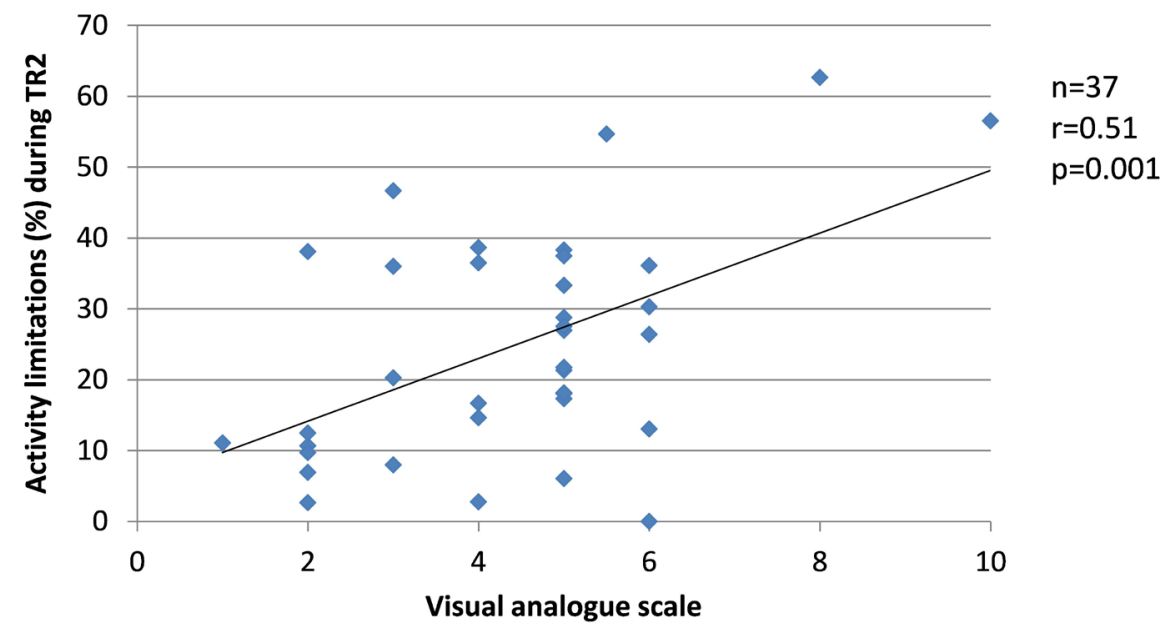

Figure 8 Correlation between the severity of pregnancy-related lumbopelvic pain (LPP) and activity limitations in the second trimester of pregnancy (TR2). 


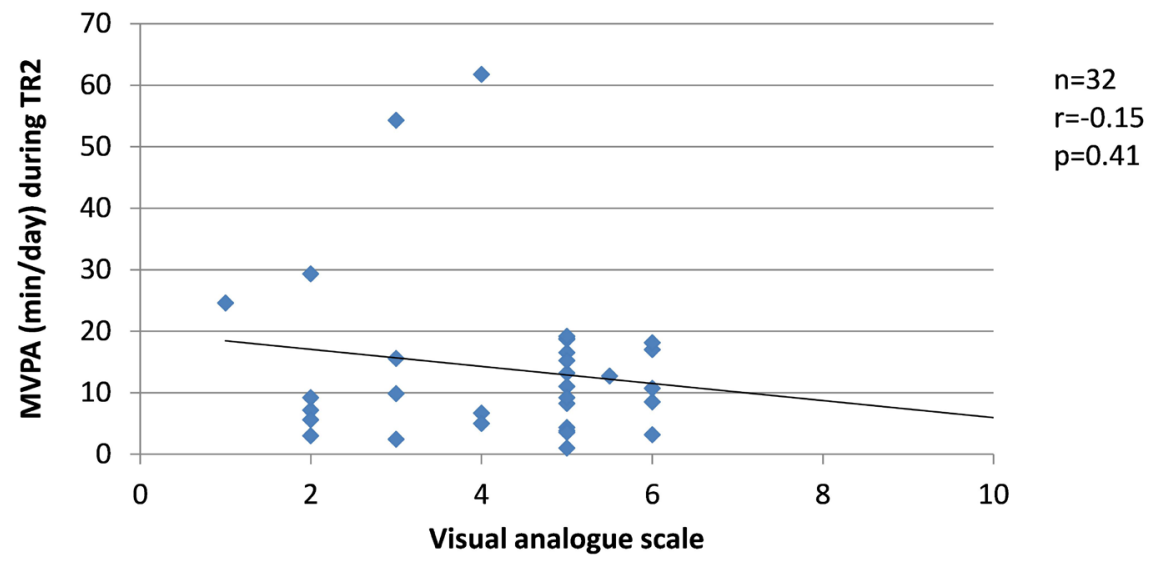

Figure 9 Correlation between the severity of pregnancy-related lumbopelvic pain (LPP) and daily moderate-to-vigorous physical activity (MVPA) in the second trimester of pregnancy (TR2).

(4/10) and were slightly anxious (35/80). Moreover, based on daily steps and physical activity recommendations, ${ }^{28} 29$ our population was considered inactive.

In our study, a total of $8(13.5 \%), 8(13.5 \%)$ and $9(15 \%)$ women removed the accelerometer to do water activities (aqua gym, swimming or bathing) during TR1, TR2 and TR3, respectively. The accelerometer was removed between one and five times during the evaluation period, and for 10 to $225 \mathrm{~min}$. Furthermore, physical activity data was missing for $10(17 \%), 7(12 \%)$ and 8 (14\%) women at TR1, TR2 and TR3, respectively, because those women did not wear the accelerometer for at least 10 hours per day for at least 4 days.

The prevalence of pregnancy-related LPP was similar in both groups during each trimester of pregnancy (TR1: $\chi^{2}=2.19, \mathrm{p}=0.33$; TR2: $\chi^{2}=2.13, \mathrm{p}=0.33$; TR3: $\chi^{2}=0.01$, $\mathrm{p}=0.92)$; the pooled prevalence increased from $42 \%$ during TR $1 \%$ to $65 \%$ during TR2\% to $68 \%$ during TR3 $\left(\chi^{2}=8.45 ; \mathrm{p}=0.01\right)$ (figure 1). Among women presenting with pregnancy-related LPP at one time point during pregnancy $(\mathrm{n}=44,26 \mathrm{SP}$ and $18 \mathrm{FT})$, pain severity significantly increased over the course of pregnancy in both groups (time effect: $\mathrm{F}=14.81, \mathrm{p}<0.0001$. figure 2), with pain severity being significantly higher during TR2 and TR3 compared with TR1. Trait anxiety decreased over the course of pregnancy in both groups (time effect: $\mathrm{F}=3.93, \mathrm{p}<0.03$. figure 3 ), with lower levels during TR2 compared with TR1, whereas state anxiety did not significantly change (figure 4). Finally, activity limitations associated with pregnancy-related LPP increased (time effect: $\mathrm{F}=18.82, \mathrm{p}<0.0001$. figure 5) whereas daily steps decreased over the course of pregnancy in both groups (time effect: $\mathrm{F}=16.03, \mathrm{p}<0.0001$. figure 6 ). The only time by group interaction effect was found for daily MVPA (time effect: $\mathrm{F}=13.11, \mathrm{p}<0.0001$; time*group interaction effect: $F=3.38, p=0.04$. figure 7 ), with daily MVPA being lower in TR3 compared with TR1 and TR2 only in SP women.

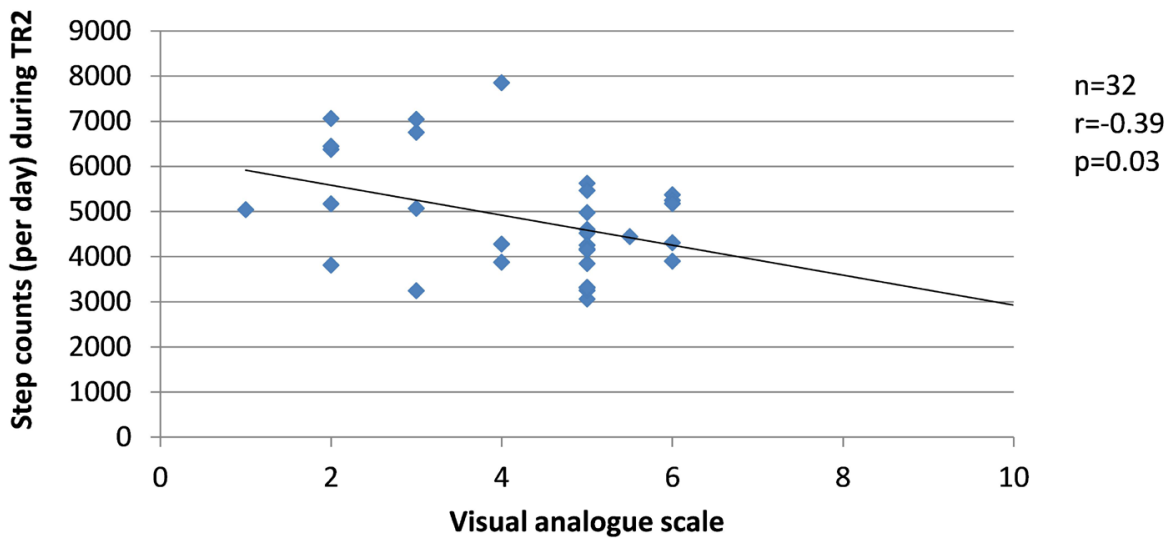

Figure 10 Correlation between the severity of pregnancy-related lumbopelvic pain (LPP) and daily step counts in the second trimester of pregnancy (TR2). 


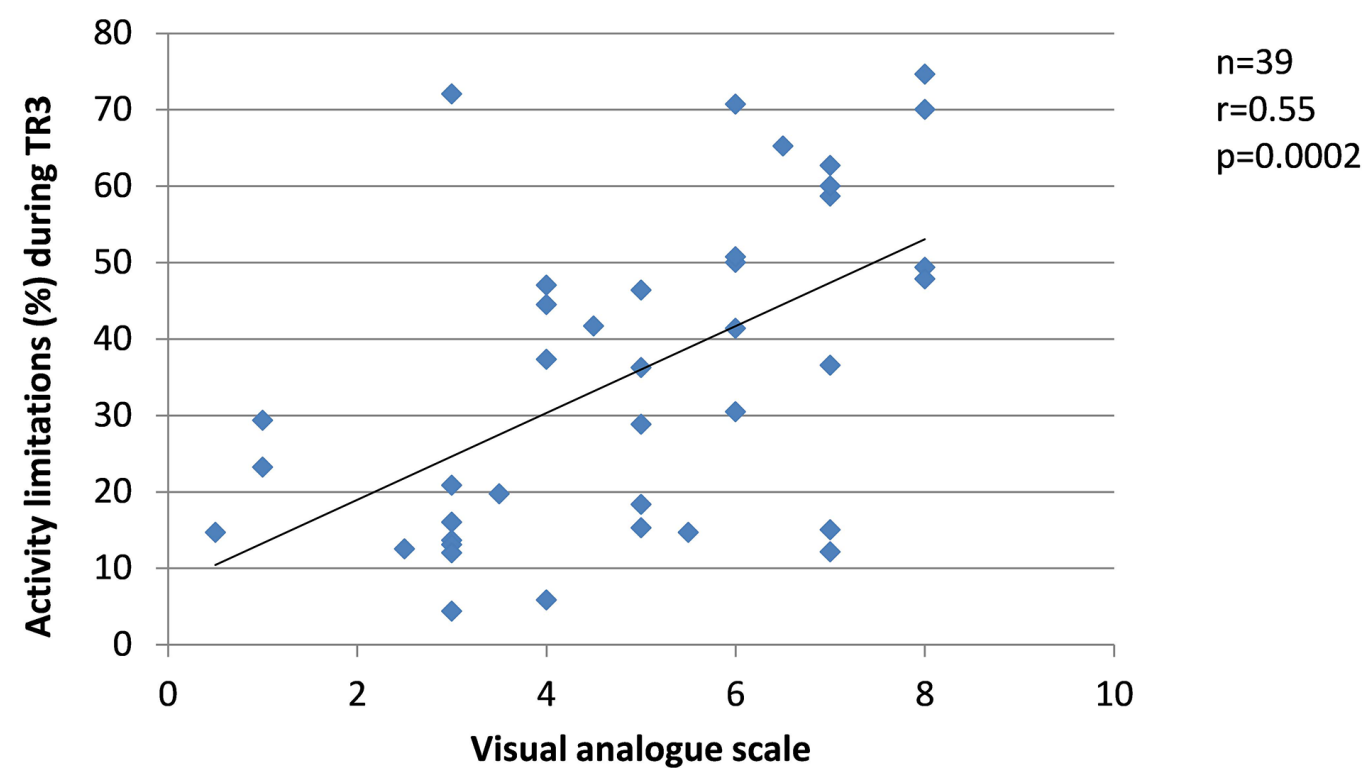

Figure 11 Correlation between the severity of pregnancy-related lumbopelvic pain (LPP) and activity limitations in the third trimester of pregnancy (TR3).

Since changes in the severity of pregnancy-related LPP, levels of anxiety and physical activity behaviours were similar between the groups, the results from SP and FT women were pooled in the correlation analyses. Among women who presented with pregnancy-related LPP, no correlation was found during TR1 between the severity of pregnancy-related LPP and anxiety or physical activity levels. During TR2, the severity of pregnancy-related LPP was positively correlated with activity limitations $(r=0.51$, $\mathrm{p}=0.001$, figure 8$)$ but negatively with daily steps $(\mathrm{r}=-0.39$, $\mathrm{p}=0.03$, figure 9 ). No correlation was found with daily MVPA (figure 10). During TR3, we found a positive correlation between the severity of pregnancy-related LPP and activity limitations $(r=0.55, p=0.0002$, figure 11$)$ and a negative correlation with daily MVPA $(\mathrm{r}=-0.41$, $\mathrm{p}=0.02$, figure 12 ). No correlation was found with daily steps (figure 13).

\section{DISCUSSION}

To the best of our knowledge, this is the first cohort study assessing the course of pregnancy-related LPP prevalence and severity in pregnant women who conceived naturally and after fertility treatments, and also looking into a possible association with anxiety levels and physical activity behaviours. Overall, our primary results showed no differences in LPP prevalence and severity, or anxiety levels between women who achieved a pregnancy naturally or after fertility treatments. As expected, the prevalence and severity of LPP increased over the course of pregnancy and were of similar magnitude than that reported in previous studies. ${ }^{10} 18$ Anxiety levels decreased from early to mid-pregnancy and were not correlated to the severity of LPP.

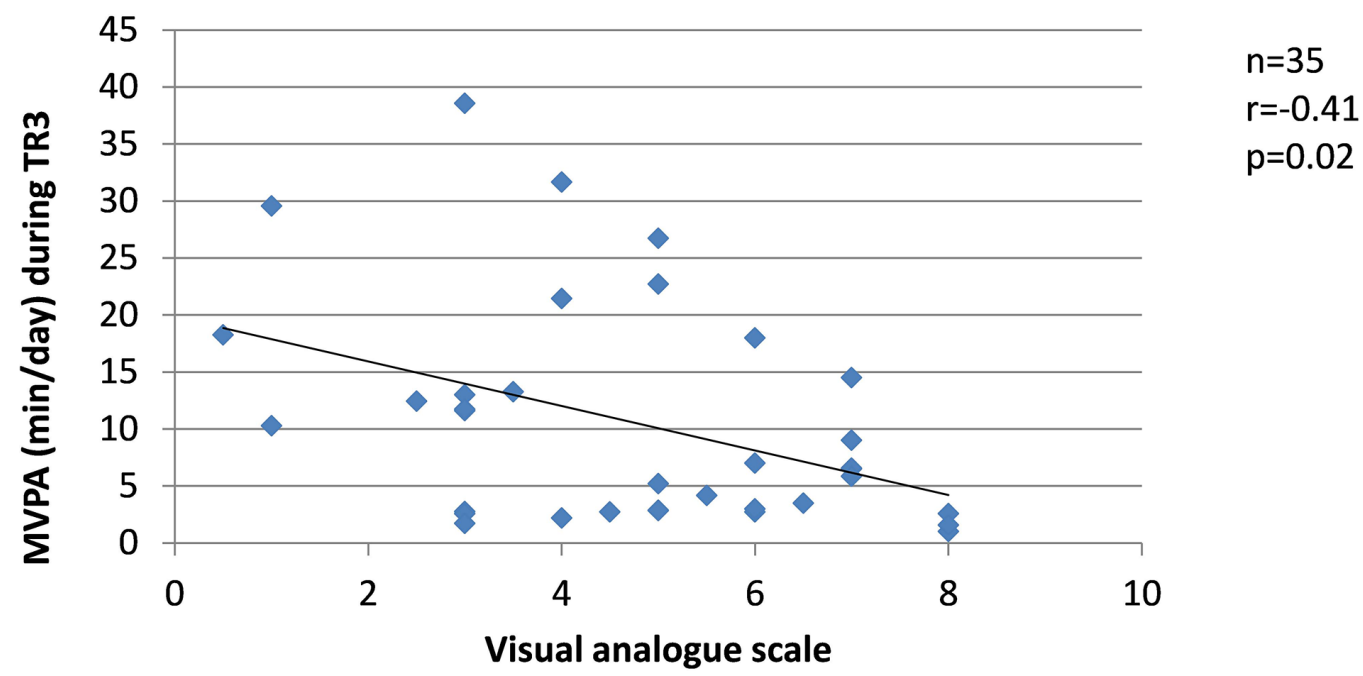

Figure 12 Correlation between the severity of pregnancy-related lumbopelvic pain (LPP) and daily moderate-to-vigorous physical activity (MVPA) in the third trimester of pregnancy (TR3). 


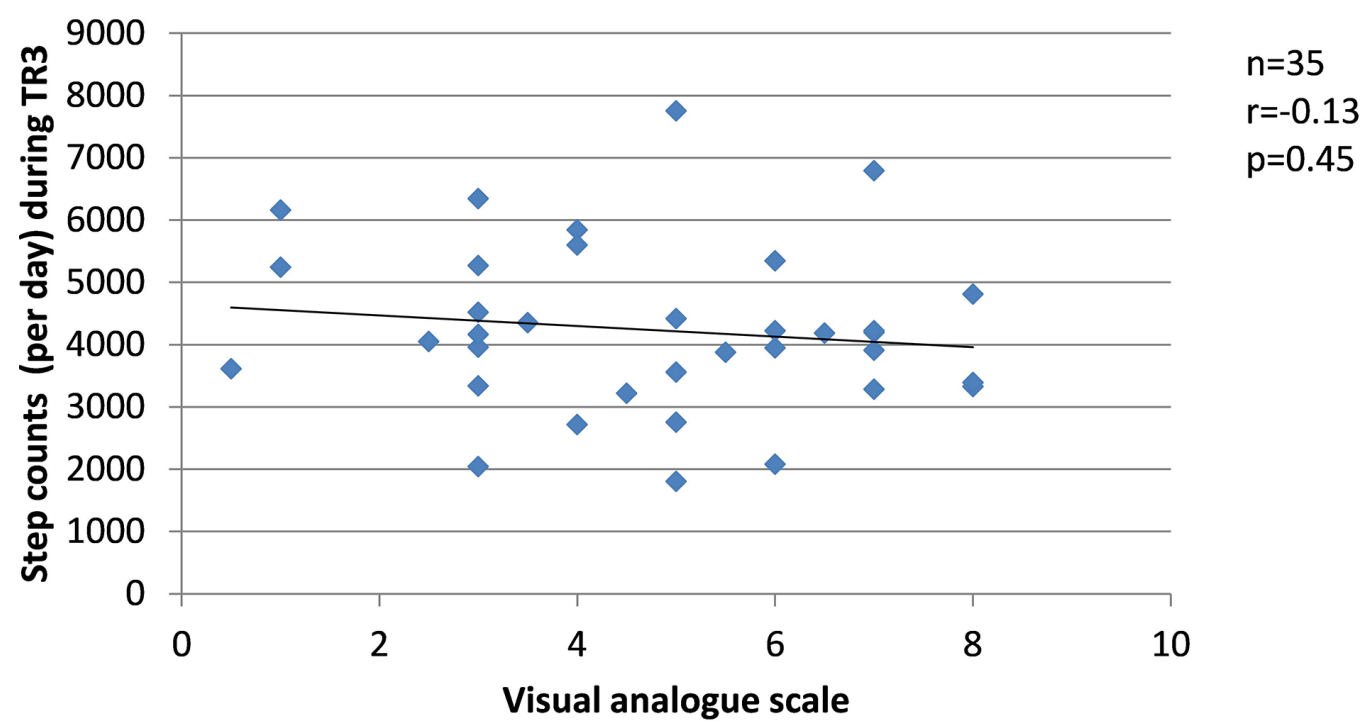

Figure 13 Correlation between the severity of pregnancy-related lumbopelvic pain (LPP) and daily step counts in the third trimester of pregnancy (TR3).

Only one study examined the evolution of the prevalence and severity of pregnancy-related PGP (PPGP) according to the mode of conception. ${ }^{15}$ This study was conducted in 31 women who conceived after IVF and 200 women who conceived spontaneously and assessed PGP at 12, 24 and 34 weeks of pregnancy. The authors found an increase in PPGP prevalence and severity over the course of pregnancy in all women, as we and other authors did. ${ }^{30}$ However, they reported a two times higher rate of PPGP in early and late pregnancy in women who achieved a pregnancy after IVF compared with those who achieved a pregnancy naturally but similar severity of PPGP. ${ }^{15}$ Importantly, many IVF women carried multiple pregnancies in that study. Given that relaxin levels are higher after $\mathrm{IVF}^{16}$ and that the number of fetuses is higher after IVF, and given that the mechanical load is higher in twin pregnancies, it is difficult to establish what causes higher rates of PPGP after IVF in this previous study.

Our hypothesis was that higher anxiety levels reported in women who conceived after fertility treatments ${ }^{17}$ would contribute to higher pregnancy-related LPP prevalence and severity in this population of pregnant women. However, we did not find any difference in anxiety levels between women who conceived after fertility treatments and those who conceived naturally. There are several reasons that may explain our result. First, the majority of women included in our sample conceived after ovarian stimulation (OS, $\mathrm{n}=7)$ or intrauterine insemination (IUI, $n=12$ ), whereas the majority of studies included in Gourounti's review reporting higher anxiety in women who conceived following fertility treatments were conducted in the context of IVF. ${ }^{17}$ Because the medical surveillance is more frequent and the procedure more invasive in the context of IVF, it is likely that IVF generates more anxiety than OS and IUI. This might partially explain why we found no differences in anxiety levels in our sample.
When examining anxiety levels over the course of pregnancy, we found a U-shaped curve, with a significant decrease in anxiety from TR1 to TR2 and a non-significant trend toward an increase from TR2 to TR3. These findings are similar to those of previous studies. ${ }^{31}{ }^{32}$ In contrast, whereas some studies reported higher anxiety in pregnant women with LBP or PGP, ${ }^{18} 33$ we found no correlation between anxiety levels and LPP severity. Our findings suggest that in our sample, anxiety and LPP were two independent phenomena.

Likewise, our secondary results showed no relationship between the mode of conception and physical limitations and physical activity behaviours, except for MVPA during TR3. The decrease in MVPA observed only in women who conceived naturally needs further investigation. Similar to previous studies, ${ }^{34-37}$ we found that with advancing pregnancy, physical limitations increased ${ }^{36-38}$ and physical activity behaviours decreased. ${ }^{34} 3539$ Our data further showed that the greater pregnancy-related LPP severity the greater physical limitation and lower physical activity levels in mid- and late pregnancy. These results are also in accordance with previous studies reporting decreased physical activity levels as physical limitations and LBP increase with advancing pregnancy. ${ }^{3640}$

\section{Limitations}

The strength of our study is its longitudinal design that allowed us to examine the evolution of several maternal health-related factors that are known to change over the course of pregnancy, in the context of spontaneous pregnancies and pregnancies achieved following FT. Moreover, our study adds knowledge about the relationship between pregnancy-related LPP severity and physical activity behaviours. However, the current study has limitations that should be acknowledged. First, our sample was heterogeneous with regard to fertility treatments used to achieve a pregnancy, with the majority of women having 
conceived after OS or IUI. This may explain the lower prevalence of PPGP and anxiety levels in women who conceived after fertility treatments. The low number of women who achieved a pregnancy following IVF prevented us to fully test our hypotheses and further larger studies are needed to better understand whether IVF contribute to pregnancy-related. Second, more than half of the women we recruited had a university degree, which is more than in our local population $(22.5 \%){ }^{41}$ This suggests a possible recruitment bias and limits the generalisability of our results. Third, although accelerometers provide a valid and objective measure of physical activity levels, non-waterproof accelerometers underestimate several types of physical activity, such as water activities. In our data set, several women removed the accelerometer to do water activities (aqua gym, swimming or bathing) during TR1, TR2 and TR3, suggesting that we possibly underestimated the level of physical activity of these women. We also had missing physical activity data because some women did not wear the accelerometer for at least 10 hours per day for at least 4 days. Finally, physical activity levels were assessed only for a 7 day period during each trimester of pregnancy. Given that each trimester lasts for more than a week, the data obtained and the results reported in relation to physical activity levels do not truly reflect the evolution of physical activity levels over each trimester and over the entire course of pregnancy. Nevertheless, the majority of the women stated in the daily diary that their physical activity behaviour over the 7 day period of evaluation reflected their habitual behaviours.

\section{CONCLUSION}

In conclusion, our findings suggest that maternal health-related factors, such as LPP, anxiety and physical activity behaviours, are not different in women who conceived after fertility treatments and those who conceived spontaneously. The lack of correlation between the severity of pregnancy-related LPP and anxiety levels suggests that they are two independent phenomena. The increase in LPP severity and activity limitations, and decrease in physical activity behaviours with advancing gestation, and the fact that the more severe LPP the greater activity limitations and physical inactivity in mid- and late pregnancy underline the importance of pregnancy-related LPP management to allow pregnant women performing their daily activities.

Acknowledgements The authors would like to acknowledge and thank Sophie Drouin, the coordinator of the fertility clinic, as well as the medical team who assisted with the recruitment, and all the women who participated to the project.

Contributors S-MR, MD and VB contributed to the study concept and design; EL and AS-L acquired the data; S-MR, MD and EL performed the statistical analysis and interpreted the data; EL and SMR drafted the manuscript; MD, AS-L and VB critically reviewed the manuscript for important intellectual content. All authors read and approved the final manuscript.

Funding This study was funded by a start-up grant from the Univeristé du Québec à Trois-Rivières (Institutional funds for research).

Competing interests None declared.

Patient consent Not required.
Ethics approval The study was approved by the local Research Ethics Committees (CER-2015-003 and CER-15-214-07.10)

Provenance and peer review Not commissioned; externally peer reviewed.

Data sharing statement № additional data are available.

Open access This is an open access article distributed in accordance with the Creative Commons Attribution Non Commercial (CC BY-NC 4.0) license, which permits others to distribute, remix, adapt, build upon this work non-commercially, and license their derivative works on different terms, provided the original work is properly cited, appropriate credit is given, any changes made indicated, and the use is non-commercial. See: http://creativecommons.org/licenses/by-nc/4.0/.

\section{REFERENCES}

1. Vleeming A, Albert $\mathrm{HB}$, Ostgaard $\mathrm{HC}$, et al. European guidelines for the diagnosis and treatment of pelvic girdle pain. Eur Spine $J$ 2008;17:794-819.

2. van Tulder M, Becker A, Bekkering T, et al. Chapter 3. European guidelines for the management of acute nonspecific low back pain in primary care. Eur Spine J 2006;15 Suppl 2:s169-s191.

3. Wu WH, Meijer OG, Uegaki K, et al. Pregnancy-related pelvic girdle pain (PPP), I: Terminology, clinical presentation, and prevalence. Eur Spine J 2004;13:575-89.

4. Gutke A, Ostgaard HC, Oberg B. Pelvic girdle pain and lumbar pain in pregnancy: a cohort study of the consequences in terms of health and functioning. Spine 2006;31:E149-55.

5. Mogren IM, Pohjanen Al. Low back pain and pelvic pain during pregnancy: prevalence and risk factors. Spine 2005;30:983-91.

6. Kanakaris NK, Roberts CS, Giannoudis PV. Pregnancy-related pelvic girdle pain: an update. BMC Med 2011;9:15.

7. Olsson C, Nilsson-Wikmar L. Health-related quality of life and physical ability among pregnant women with and without back pain in late pregnancy. Acta Obstet Gynecol Scand 2004;83:351-7.

8. Elden $\mathrm{H}$, Lundgren I, Robertson E. Life's pregnant pause of pain: pregnant women's experiences of pelvic girdle pain related to daily life: a Swedish interview study. Sex Reprod Healthc 2013;4:29-34.

9. Mogren I. Perceived health, sick leave, psychosocial situation, and sexual life in women with low-back pain and pelvic pain during pregnancy. Acta Obstet Gynecol Scand 2006;85:647-56.

10. Wang SM, Dezinno P, Maranets I, et al. Low back pain during pregnancy: prevalence, risk factors, and outcomes. Obstet Gynecol 2004;104:65-70.

11. Owe KM, Nystad W, Bo K. Correlates of regular exercise during pregnancy: the norwegian mother and child cohort study. Scand J Med Sci Sports 2009;19:637-45.

12. Mudd LM, Owe KM, Mottola MF, et al. Health benefits of physical activity during pregnancy: an international perspective. Med Sci Sports Exerc 2013;45:268-77.

13. Davies GA, Wolfe LA, Mottola MF, et al. Exercise in pregnancy and the postpartum period. J Obstet Gynaecol Can 2003;25:516-29.

14. ACOG Committee Opinion. Physical activity and exercise during pregnancy and the postpartum period. Obstet Gynecol 2015;126:e135-42.

15. Kristiansson P, Nilsson-Wikmar L, von Schoultz B, et al. Back pain in in-vitro fertilized and spontaneous pregnancies. Hum Reprod 1998;13:3233-8.

16. Kristiansson P, Svärdsudd K, von Schoultz B, et al. Supraphysiological serum relaxin concentration during pregnancy achieved by in-vitro fertilization is strongly correlated to the number of growing follicles in the treatment cycle. Hum Reprod 1996;11:2036-40.

17. Gourounti K. Psychological stress and adjustment in pregnancy following assisted reproductive technology and spontaneous conception: A systematic review. Women Health 2016;56:1-21.

18. Kovacs FM, Garcia E, Royuela A, et al. Prevalence and factors associated with low back pain and pelvic girdle pain during pregnancy: a multicenter study conducted in the Spanish National Health Service. Spine 2012;37:1516-33.

19. Girard MP, Marchand AA, Stuge B, et al. Cross-cultural adaptation of the pelvic girdle questionnaire for the french-canadian population. J Manipulative Physiol Ther 2016;39:494-9.

20. Ostelo RW, Deyo RA, Stratford P, et al. Interpreting change scores for pain and functional status in low back pain: towards international consensus regarding minimal important change. Spine 2008;33:90-4.

21. Gauthier J, Bouchard S. Adaptation canadienne-française de la forme révisée du state-trait anxiety inventory de spielberger. Can $J$ Behav Sci 1990;25:559-78. 
22. Spielberger CD. Manual for the State-Trait Anxiety Inventory (Form Y. Palo Alto: Consulting Psychologist Press, 1983.

23. Gunning MD, Denison FC, Stockley CJ, et al. Assessing maternal anxiety in pregnancy with the State-Trait Anxiety Inventory (STAI): issues of validity, location and participation. J Reprod Infant Psychol 2010;28:266-73.

24. Stuge B, Garratt A, Krogstad Jenssen H, et al. The pelvic girdle questionnaire: a condition-specific instrument for assessing activity limitations and symptoms in people with pelvic girdle pain. Phys Ther 2011;91:1096-108.

25. Colley RC, Garriguet D, Janssen I, et al. Activité physique des adultes au Canada: résultats d'accélérométrie de l'Enquête Cannadienne sur les mesures de la santé de 2007-2009. Statistique Canada Rapports sur la santé 2011.

26. Freedson PS, Melanson E, Sirard J. Calibration of the Computer Science and Applications, Inc. accelerometer. Med Sci Sports Exerc 1998;30:777-81.

27. Harrison $\mathrm{CL}$, Thompson RG, Teede HJ, et al. Measuring physical activity during pregnancy. Int $J$ Behav Nutr Phys Act 2011;8:19.

28. Tudor-Locke C, Bassett DR. How many steps/day are enough? Preliminary pedometer indices for public health. Sports Med 2004;34:1-8.

29. Evenson KR, Mottola MF, Owe KM, et al. Summary of international guidelines for physical activity after pregnancy. Obstet Gynecol Surv 2014;69:407-14.

30. Kristiansson P, Svärdsudd K, von Schoultz B. Back pain during pregnancy: a prospective study. Spine 1996;21:702-9.

31. Lee AM, Lam SK, Sze Mun Lau SM, et al. Prevalence, course, and risk factors for antenatal anxiety and depression. Obstet Gynecol 2007;110:1102-12.

32. Teixeira C, Figueiredo B, Conde A, et al. Anxiety and depression during pregnancy in women and men. $J$ Affect Disord 2009;119:142-8.
33. Elden H, Gutke A, Kjellby-Wendt G, et al. Predictors and consequences of long-term pregnancy-related pelvic girdle pain: a longitudinal follow-up study. BMC Musculoskelet Disord 2016;17:276.

34. Evenson KR, Wen F. Prevalence and correlates of objectively measured physical activity and sedentary behavior among US pregnant women. Prev Med 2011;53:39-43.

35. Rousham EK, Clarke PE, Gross H. Significant changes in physical activity among pregnant women in the UK as assessed by accelerometry and self-reported activity. Eur J Clin Nutr 2006;60:393-400.

36. Cramp AG, Bray SR. A prospective examination of exercise and barrier self-efficacy to engage in leisure-time physical activity during pregnancy. Ann Behav Med 2009;37:325-34.

37. Robinson HS, Veierød MB, Mengshoel AM, et al. Pelvic girdle pain-associations between risk factors in early pregnancy and disability or pain intensity in late pregnancy: a prospective cohort study. BMC Musculoskelet Disord 2010;11:91.

38. Chang HY, Lai YH, Jensen MP, et al. Factors associated with low back pain changes during the third trimester of pregnancy. J Adv Nurs 2014;70:1054-64.

39. Downs DS, LeMasurier GC, DiNallo JM. Baby steps: pedometerdetermined and self-reported leisure-time exercise behaviors of pregnant women. J Phys Act Health 2009;6:63-72.

40. Poston L, Briley AL, Barr S, et al. Developing a complex intervention for diet and activity behaviour change in obese pregnant women (the UPBEAT trial); assessment of behavioural change and process evaluation in a pilot randomised controlled trial. BMC Pregnancy Childbirth 2013;13:148.

41. Statistics Canada. Labour Force Survey, special compilation, adapted by the Institut de la statistique du Québec. 2017. 